\title{
Urgences
}

\section{Guy Lafleur : sous le signe des soleils \\ Georges-Hébert Germain, Guy Lafleur. L'ombre et la lumière , Montréal, Art Global/Lilbre expression, 1990.}

\section{Renald Bérubé}

Numéro 29, octobre 1990

Éclats d'œuvre

URI : https://id.erudit.org/iderudit/025616ar

DOI : https://doi.org/10.7202/025616ar

Aller au sommaire du numéro

Éditeur(s)

Urgences

ISSN

0226-9554 (imprimé)

1927-3924 (numérique)

Découvrir la revue

Citer ce compte rendu

Bérubé, R. (1990). Compte rendu de [Guy Lafleur : sous le signe des soleils / Georges-Hébert Germain, Guy Lafleur. L'ombre et la lumière, Montréal, Art Global/Libre expression, 1990.] Urgences, (29), 101-105.

https://doi.org/10.7202/025616ar d'utilisation que vous pouvez consulter en ligne.

https://apropos.erudit.org/fr/usagers/politique-dutilisation/ 


\section{deliveline}

\section{Guy Lafleur: sous le signe des soleils}

\section{d Bernard Boucher}

Comme Robert Charlebois qui, lors d'un super show au Stade olympique, avait demandé à la foule:

"L'aimez-vous, Guy Lafleur? *

La foule s'était mise à hurler. Charlebois avait ajouté:

- Moi aussi, je l'aime. L'hiver, il remplace le soleil."

\section{Georges-Hébert} Germain 1

Guy Lafleur, tu étais la chaleur de notre hiver intérieur, c'était toi qui resserrais les liens invisibles de la collectivité, toi le rédempteur, mihomme mi-démon blond, portant le sens de notre combat national sur LA SCÉNE SPORTNE, sur la glace éblouissante cormme sur les fcrans scintillants, là où la victoire nous était enfin concédés.

Jean-Pierre April ${ }^{2}$

La pratique sportive, on le sait, peut se lire comme une vaste métaphore. Comme un micro- cosme de l'univers plus global, comme un lieu circonscrit où se joue d'une manière particulière le theatre du monde, comme une mise en abyme hautement vedettarisée d'un monde tout aussi spectaculairement médiatise. Les athlètes et les comédiens pratiquent le mème métier: ce sont des joueurs, et nous jouons tous et toutes notre role.

Vaste métaphore, mise en abyme hypercondengee de ce qu'il est convenu d'appeler la destinée humaine: le temps sportif, celui du match ou de la carrière de l'athlete, est de brève durée; d'où la nécessité d'un apprentissage accéléré avant le déclin qui guette, et alors que le risque de blessures est constant, l'adversaire impitoyable, les spectateurs immensément exigeants puisqu'ils ont bien souvent a l'athlete ou son equipe délégué leurs rêves et leurs aspirations - dans le temps sportif, le passage de héros à bouc émissaire (et vice versa) peut s'effectuer en une fraction de seconde.

Vaste métaphore et mise en abyme hypercondensé de tel mode de vie socio-économique aussi: les athletes nord-américains connaissent bien les vertus (?) de rentabilité du monde du spectacle, quel qu'il soit. Et pourquoi done y a-t-il tant d'arbitres ou de juges divers pour un match de baseball, de football américain ou de hockey, et un seul dans les matches que nous appelons de soccer? Sans doute, pourrait-on dire, l'apprentissage de l'Autorité ne s'est-il pas effec- 
tué selon les mêmes modes ni les mêmes traditions en Amérique et en Europe.

On comprendra dès lors que le journaliste sportif / écrivain americain Roger Kahn intitule "The Metaphor of Sport " la première partie d'un ouvrage dont le titre est How the Weather Was ${ }^{3}$, titre qui provient lui-même d'un extrait de Hemingway donné en épigraphe à louvrage; et que le poète américain/ amateur de sports (surtout du baseball) Donald Hall s'intéresse particulièrement au rôle de la métaphore dans l'écriture sportive dans son livre intitulé Fathers Playing Catch with Sons et sous-titre Essays on Sport (Mostly Baseball) 4 . Le sport n'est certes pas une maladie - a moins que la personne qui le pratique ou celle qui le consomme à un titre ou a un autre ne devienne joueur selon le titre du roman de Dostolevski ou à la Pete Rose. N'empeche que l'intitule * The Metaphor of Sport * de Kahn peut aussi renvoyer le lecteur à un autre intitulé, celui de l'ouvrage de Susan Sontag, Illness as Metaphor ${ }^{5}$. Comment dire? La maladie, notre crainte a tous et toutes; le sport, l'un des lieux par excellence ou projeter, tous et toutes, nos aspirations et nos rêves. L'être humain est Homo ludens, selon le titre du livre de Huizinga 6 - nous jouons toujours, la vie comme le reste - 8 iil reste quelque chose hors la vie -, a qui perd gagne. Et il tombe obligatoirement sous le sens, malgre le cour ou la raison qui ont chacun et chacune leurs raisons, que vivre, nolens volens, se revèle toujours mortel.

Comme il doit malgre tout tomber sous le sens, cela étant écrit ou lu, qu'il s'agit bien ici, étant donné l'intitulé de ce texte et sa première épigraphe, de Guy Lafleur et de la très belle biographie que lui a consacré GeorgesHébert Germain. Biographie qui n'est pas la première à etre consacré à Guy Lafleur ?; biographie qui, à sa manière, pose la problématique du genre (auto)biographique que nous avons déjà soulevée ailleurs 8 ; quand une vedette se raconte tout autant qu'elle est racontée, qui donc raconte, qui donc écrit? Ce qui est sûr par ailleurs, c'est la qualité de ce qui est ici raconté le gros livre de Germain, malgre quelques erreurs qu'auront vite repérées les amateurs de hockey ${ }^{9}$, se révèle d'une écriture bien soutenue, qui sacrifie rarement a l'ampoule ou au cliche; d'un intérêt réel qui ne sacrifie pas la mode potineuse ou statisticienne. Et l'énorme succès de librairie qui a salué la parution de l'ouvrage doit bien témoigner de cette qualité, comme il témoigne aussi et sans doute aucun de l'énorme emprise que Guy Lafleur, qui aura mis du temps à la reconnaître pleinement et qui en demeure toujours un peu étonné sinon intimidé, exerce sur la population québécoise.

Car si L'ombre et la lumière est la biographie du Guy Lafleur, elle ne saurait faire l'́conomie des faits suivants: Guy Lafleur est un joueur de hockey et le hockey est un sport d'équipe. Or l'equipe avec laquelle s'est surtout illustré Lafleur s'appelle les Canadiens (selon le sens qu'avait ce nom au début du siècle) et est aussi surnommé la Sainte-Flanelle; son lieu de résidence est le Forum de Montréal, aussi surnommé la Mecque du hockey. On le sait, les Canadiens ont longtemps été l'Institution privilégiée sinon unique de nos succès - il est assez clair, du point de vue même 
de l'histoire, que l'émeute du Forum, qu'engendra en 1955 la suspension de Maurice Richard, peut se lire comme un avant-texte, un signe avant-coureur de la Révolution tranquille. S'agissant de Guy Lafleur, L'ombre et la lumiere doit aussi parler des Canadiens, equipe de joueurs de hockey ayant le statut d'Institution, d'Organisation selon le mot ironico-mafieux utilisé par Germain, et des supporters qui appuient Lafleur et/ou les Canadiens. Existe-t-il tel genre que la biographie collective, c'est-à-dire que l'étude d'une collectivité et de lune de ses institutions menée à loccasion de la biographie de l'un de ses héros?

Retour à la métaphore: car Guy Lafleur est un Héros. Il l'était déjà dans les jeunes dix ans de ses áges alors qu'il devenait la vedette adulée du Tournoi international pee wee de Quevec au début des années 1960; il le sera encore par ses exploits de joueur d'âge junior qui créeront les Remparts de Québec et obligeront le rusé Sam Pollock des Canadiens, Renard (selon Germain) ou Ulyse de toutes les astuces, à de mystifiantes manceuvres visant a permettre aux Canadiens de "repecher * Lafleur a la fin de son age junior; il le sera bien sur a l'age de sea grandes performances avec les Canadiens, alors qu'il dominait, grand maftre du patin, de la vitesse et de la feinte, la Ligue nationale du hockey. Et il saura l'etre aussi a loccasion des moments difficiles ou imprevus: a loccasion de sa retraite - de $8 \mathrm{a}$ mise a la retraite par l'Organisation: Serge Savard et Jacques Lemaire ne sortent certes pas grandis du livre de Germain puis de son retour au jeu, alors même qu'il était élu, joueur retraite, au Panthéon de la Renom- mée du hockey, ce qui rend le hockeyeur aussi immortel que l'appartenance académie française pour l'écrivain. Statut particulier ou exceptionnel: Guy Lafleur est un Immortel qui joue encore et toujours au hockey. La science-fiction de la nouvelle de Jean-Pierre April avait vu juste! Le mythe, la légende ou la métaphore ne sauraient demander davantage.

Jeune hockeyeur dans les dix ans de ses ages, Lafleur avait une idole: Jean Beliveau, grande vedette à Québec d'abord, puis à Montréal. Choix signifiant et judicieux: à sa façon, Lafleur sera, sur les patinoires, l'artiste elégant et virtuose que Beliveau avait eté, et dont la rapide chronologie sportive faisait de toute manière le successeur. Mais il n'y a pas que le hockeyeur, il y a aussi la personne Guy Lafleur, ses attitudes et son style. Chez qui dominent les valeurs de passion perfectionniste, c'est-àdire de détermination farouche et de franchise a la fois nalve et sans compromis. En quoi Guy Lafleur allait prendre la releve de Maurice Richard, de LA légende de LA SCENE SPORTIVE quebecoise, pour parler (écrire?) comme April qui lui-meme, nolens volens, nous renvoie a Octave Mannoni 10. Questions qui pourtant demeurent en suspens méme après la lecture du livre de Germain : comment expliquer les difficiles premières années de Guy Lafleur lors de son arrivee chez les Canadiens, comment expliquer ses nombreuses blessures et sa soudaine baisse de régime lors de ses dernières années chez les mêmes Canadiens? Il me semble parfois que se manifeste la comme une volonté entetee et perfectionniste d'etre soi-meme selon soi, d'etre 
his own man: je serai ce que je suis et selon mon propre mode d'étre, qui ne renvoie pas nécessairement à ce que l'on attend de moi. Je serai Lafleur, je serai le meilleur ou ne serai pas comme Béliveau fut Béliveau, comme Gretzky (qui allait succéder à Lafleur comme grand maitre de la Ligue nationale de hockey) allait etre Gretzky. Le perfectionnisme, dans l'univers du spectacle vedettarisé en particulier, peut aussi se lire comme une forme de protestation: etre ou ne pas etre, jouer ou ne pas jouer - selon ses propres criteres d'identité.

Biographie ou autobiographie: le sous-titre du livre de Germain, L'ombre et la lumière, provient d'un poème de Lafleur à son épouse et intitulé *Chandelle (que disent donc les manes de Bachelard?), poème sur lequel s'achève le livre. Et Germain sait s'amuser qui écrit, au début des dédicaces de son livre: "Je dois une fière chandelle...* Comme il sait aussi, journaliste choisi par Lafleur pour ecrire sa biographie, rendre un hommage considérable à l'épouse de ce dernier: « Je dédie ce livre à Lise Lafleur qui croit que toute vérité est bonne à dire". Vérité: sous quel autre signe peut donc se situer une biographie? Et il est plaisant de constater que L'ombre et la lumière a été publié chez Art Global/Libre Expression: qu'est donc l'art, celui du hockeyeur comme celui de l'écrivain, sinon le lieu de la libre expression de l'artiste?

Dans le monde des étoiles de la scène, Guy Lafleur aura vécu (et vit encore) sous tous les éclairages. Le mérite du livre de Germain, dans ses divisions appropriées en périodes, entractes et prolongations, réside dans sa volonté non pas d'éblouir, mais de ne ménager aucun desdits éclairages: démon blond, Guy Lafleur vit sous le soleil du jour, sous le soleil de minuit et sous les feux de la rampe que sont les soleils des projecteurs.

\section{Renald Bérubé}

1 Georges-Hébert Germain, Guy Lafleur. L'ombre et la lumiere, Montréal, Art Global/Libre Expression, 1990, p. 223.

2 Jean-Pierre April, - Le Fantôme du Forum ", nouvelle parue d'abord dans Imagine... Revue de science-fiction quebecoise, Montréal, $n^{\circ} 7$, mars 1981, p. 29-47, [p. 45]; puis reprise dans le livre de JeanMarc Gouanvic (Dix nouvelles de science-fiction réunies et présentées par), Les années-lumiere, Montreal, VLB, 1983, p. 49-50.

3 Roger Kahn, How the Weather Was, Signet Book, 1973; Kahn, on le sait, est aussi l'auteur du superbe livre The Boys of Summer publié en 1972, dans lequel le sport joue un rôle métaphorique fort important. Nous avons présente un bref compte rendu de The Boys of Summer ici meme dans Urgences, $n^{\circ} 15$, octobre 1986, p. 112-113.

4 Donald Hall, Fathers Playing Catch with Sons. Essays on Sport (Mostly Baseball), New York, Laurel Book, 1986, 198 p. Lire en particulier: \& Proseball : Sports, Stories and Style *, p. 111-141.

5 Susan Sontag, Illness as Metaphor, New York, Farrar, Straus and Giroux, 1978, $88 \mathrm{p}$.

6 Johan Huizinga, Homo ludens (Essai sur la fonction sociale du jeu), coll. * Les Essais *, Paris, Gallimard, 1977, 341 p.

7 Parue douze ans avant celle qui nous intéresse, la biographie ecrite par le journaliste sportif Claude Larochelle, Guy Lafleur. Le Demon blond, Québec, Lotographie inc., 1978,250 p. Et le pere de Guy Lafleur a aussi publié un livre consacré à son fils, livre évoqué par Georges-Hébert Germain.

8 Lors d'une communication intitulee * De la pratique sportive de 
l'autobiographie * présenté à l'Universite Laval le 29 mai 1989 dans le cadre du congres annuel des Societés savantes.

9 Par exemple, Sam Pollock ne pouvait *jeter son dévolu * sur Gilbert Perreault (p. 172); et Terry Harper lui-meme doit bien etre tout émerveillé de se retrouver dans la phrase suivante: « Enfin, les journalistes avaient cegse de commenter ses moindres faits et gestes, de le [Guy Lafleur] prendre pour un measie et de le comparer a Béliveau, orr, a Geofirion, a Harper, avx Richard, a Hull et compagnie ! * (p. 196197).

10 Octave Mannoni, Clefs pour l'Imaginaire ou lautre scène, coll. * Le champ freudien *, 1969, Paris, Seuil, 322 p.. Il convient que les notes, dans un article consacré a Guy Lafleur, s'arretent au numéro 10.

\section{Mario Luzi, Cahier gothique, traduit de Pitalien par Jean-Yves Masson, coll. "Terra d'altri», Lagrasse, Verdier, 1989, $144 \mathrm{p}$.}

Vaste gouffre apporté dans

lamas de la brume

Par l'irrascible vent des mots qu'il n'a pas dits,

Le néant à cet Homme aboli de jadis:

- Souverirs d"horizons, qu'estce, o toi, que la terre?

Hurle ce songe; et, voix de la clarté s'altère,

L'espace a pour jouet le cri: - Je ne sais pas!"

Stéphane Mallarmé

C'est Verdier, l'éditeur, qui m'a fait découvrir Mario Luzi, cet obscur inconnu chez nous au Québec, ailleurs je ne sais. C'est dans le rayon "poésie" de ma librairie préférée que j'ai remarqué l'objet. Le livre était beau, comme tout ce que fait Verdier en général.

Mario Luzi est né près de Florence en 1914. Il est considéré comme l'un des grands poètes italiens du siècle. Il est également critique et théricien de la littérature. Je ne sais s'il vit encore; nous savons cependant qu'en 1989, il révisa la traduction en français des textes que l'on retrouve dans Cahier gothique. Mario Luzi a fait paraître en traduction française plus de sept titres chez divers éditeurs dont Verdier et Flammarion.

Cahier gothique parut en italien en 1960 sous le titre original de Un brindisi, Quaderno gotico. Pour la traduction française, il aura fallu attendre en 1989. Cette traduction, soit dit en passant exceptionnelle, est de Jean-Yves Masson. Majoritairement, les textes de ce recueil ont été écrits dans les années quarante, années de guerre et de fin du monde.

Cahier gothique se divise en deux grandes parties qui furent éditées séparément a l'origine. D'abord Une libation qui elle. même se divise en quatre parties, puis Cahier gothique qui est composé de quatorze textes numérotés en chiffres romains. Nous pouvons des lors remarquer dans sa structure la récurrence du chiffre quatre qui se retrouve également implicitement dans la lettre du titre du recueil: Cahier. On définit *cahier " comme un assemblage de quatre feuilles cousues ou pliées ensemble et munies d'une couverture. "Cahier * vient du latin 